\title{
Association between sleep pattern and body mass index among undergraduate health colleges' students at Qassim University, Saudi Arabia
}

\author{
Fadyah Ali Alfarhan*1, Masheal Al.Matrouk ${ }^{2}$, Haifa AIGaowba ${ }^{3}$, Raghad AlHamely ${ }^{1}$, Hanan M. Tork ${ }^{4}$ \\ ${ }^{1}$ King Faisal Specialist Hospital \& Research Center, Riyadh, Saudi Arabia \\ ${ }^{2}$ Sulaiman Alhabib Hospital, Qassim, Saudi Arabia \\ ${ }^{3}$ Buraydah Central Hospital, Qassim, Saudi Arabia \\ ${ }^{4}$ College of Nursing, Qassim University, Saudi Arabia
}

Received: January 5, 2018

DOI: $10.5430 /$ jnep.v8n8p86
Accepted: February 26, 2018

Online Published: March 28, 2018

URL: https://doi.org/10.5430/jnep.v8n8p86

\begin{abstract}
Background and objective: Better quality and adequate amount of sleep are essential to have better cognitive performance and by contrast, poor bedrest is related to a diminishing of cognitive and psychological functioning and deteriorating physical health. Recently the quality of sleep among various segments of the population has become a focus of continuous research and investigations, where insufficient sleep may lead to adverse cardio-metabolic effects by influencing body weight, blood pressure, and glucose tolerance. The objective of this study is to explore the associations between sleep patterns and Body Mass Index (BMI) among undergraduate female students in health colleges.

Methods: Across-sectional study was utilized among 288 of undergraduate female students who represent $30 \%$ of all undergraduate students in health Colleges of female section, Qassim University. The data regarding their sleeping pattern was collected and calculated by using a validated self-report questioner (Pittsburgh Sleep Quality Index) and Body Mass.

Results: According to PSQI scoring, both categories good and poor sleep maximum according to PSQI scoring; both categories good sleep and poor sleep maximum participant's BMI was normal but no statistically significant association was seen between BMI of poor sleep was higher in overweight and obese participants from all departments and also no statistically significant association was seen between poor sleep and body mass index of participant As for the sleep quality as a single question and sleep duration in relation to BMI for different specialties, only for nursing students there was a statistically significant difference of sleep quality/duration in relation to their BMI.

Conclusions: Frequency of poor sleep was higher in overweight and obese participants but as per findings there was no statistically significant association was detected between PSQI score and BMI of participants.
\end{abstract}

Key Words: Body mass index, Undergraduate students, Sleep pattern, Sleep quality

\section{INTRODUCTION}

College is a time for change, when many young adults experience self-sufficiency and freedom from direct supervision for the first time, greater academic and social stresses, and unpredictable schedules are experienced. Major conversions in lifestyle such as unstable diets, and lack of sleep can be harm-

\footnotetext{
*Correspondence: Fadyah Ali Alfarhan; Email: Alfarhan.f@hotmail.com; Address: King Faisal Specialist Hospital \& Research Center, Riyadh, Saudi Arabia.
} 
ful to the students' well-being. ${ }^{[1]}$ Many researches concede that college pupils experience frequent and severe bedtime issues that negatively effect their health and well-being. ${ }^{[2-6]}$ Sleep is a universal part of life and one of the essential behavioral components of sustaining health state.$^{[7,8]}$ Although the specific functions of sleep are still beyond a comprehensive understanding, a large body of data show it's a serious role in many important somatic, cognitive, and psychological processes. ${ }^{[9]}$

It is true that sleep is beneficial for powerful conservation and neuronal recuperation, ${ }^{[8]}$ synaptic homeostasis, and brain flexibility. For example, sleep is shown to serve many metabolic, immunity, thermoregulatory, cardio, and respiratory functions, all accountable for the normal brain and body homeostasis. ${ }^{[10,11]}$ By contrast, sleep dysfunction is linked to the development of physical and psychological issues including cardiovascular problems, diabetes, obesity, anxiety, and all-cause high rates of death ${ }^{[12]}$ and number of studies have supported that the metabolic rate related to interference of normal sleep behaviors may related to the development of obesity, cardiovascular disease, diabetes, and diabetes. ${ }^{[13-26]}$ In the current study, the authors applied crosssectional study that explore the association between Body Mass Index (BMI) and sleep pattern among undergraduate female students of health colleges in Qassim University.

\section{Sleep pattern and body mass index}

There is an increasing acknowledgment and several research findings support an association between underweight (e.g. anorexia), overweight/obesity and weight fluctuation (e.g. weight loss, weight gain) and sleep problems. ${ }^{[27]}$ Concurrent with high rates of obesity among people in the United States, there has been an alteration in sleeping manners. ${ }^{[28,29]}$ Insomnia and sleep issues have been widely studied in the larg population. ${ }^{[1]}$ The intervening processes between sleep health and BMI are complex and not well understood yet.

The BMI is generally used as a means of correlation between groups related to general mass and can serve as a basic means of estimating adiposity. However, the duality of the BMI is, that, whilst easy-to-use as a general calculation, it is limited in how accurate and pertinent the data obtained from it can be. Generally, the Index is suitable for recognizing trends within sedentary or overweight individuals because there is a smaller margin for errors. ${ }^{[30]}$ High BMI appears associated with breathing difficulties such as apnea. ${ }^{[29]}$ Around the years and following vigorous examinations of the topic, our knowledge of sleep issues has both dramatically extended and significantly changed. ${ }^{[31]}$ Better quality sleep and adequate amount of sleep are necessaryto have improvedcognitive performance and avoid many health problems and psychiatric disorders. ${ }^{[32]}$ In another side, sleep loss, long-term sleep deprivation and alterations in sleep quality are substantial problems in modern society; also sleep quality may play a role in disease etiology. ${ }^{[33]}$ Recently, the quality of sleep among various segments of the population has become a focus of continuous research and investigation. ${ }^{[34]}$ On the other hand, the prevalence of overweight and obesity, has elevated dramatically over the past decade. ${ }^{[35-37]}$ Body Mass Index (BMI) has been defined by World Health Organization $(\mathrm{WHO})$ as $\left[\mathrm{BMI}=\right.$ weight $(\mathrm{kg}) /$ height $\left._{\left(\text {meters }^{2}\right.}{ }^{2}\right]$ a widely used measure to evaluate global body fat masses. ${ }^{[38]}$ Al-Nohair ${ }^{[39]}$ illustrated that Gulf countries having the highest rate of obesity and concurrent with the epidemiological transition trend at Kingdom of Saudi Arabia (KSA); the prevalence rate of high BMI and obesity are sharply rising with approximately three-quarters of the female adult population. ${ }^{[40]}$

According to Al-Nohair, ${ }^{[39]}$ KSA represents one of the top ten countries worldwide in term of obesity. In spite of this accretion of BMI has been observed around all ages juveniles and teenagers reaching $31.8 \%$ in $2010 .{ }^{[35]}$ In the same way elevated rates have been estimated among college students; with over one-third reporting BMI $\pm 25 .^{[41-43]}$ The association between eating behavior disturbance and sleep difficulties is also documented as Night Eating Syndrome which characterized by recurrent episodes of the night eating, as manifested by eating after awakening from sleep or excessive food consumption after the evening meal. ${ }^{[44]}$ In 2010, Lombardi et al. ${ }^{[45]}$ mentioned that fatigue has been linked to adverse safety outcomes, and poor quality or decreased sleep has been associated with obesity. Moreover, objective measures of sleep showed that this pattern of disordered eating occurs during non-REM sleep and is associated with low sleep efficiency. ${ }^{[46]}$ In addition, Bjorvatn et al. ${ }^{[47]}$ illustrated that short self-reported sleep duration is linked to elevated BMI and may change appetite hormones. An inverse association between BMI and sleep difficulty and sleep duration was reported in other study which performed among University students ${ }^{[48]}$ also, among females rather than males from the general population of China. ${ }^{[49]}$

Sleep difficulties may represent a risk factor for increasing in BMI. This study may improve the knowledge regarding this public health topic in non-clinical populations, stressing the need for health professionals to pursue strategies to promote adequate sleep and eating patterns to prevent disorders from both areas. The aim of the present study is to explore the associations between sleep patterns and BMI among undergraduate female students in health colleges, at Qassim University through hypothesizing that BMI is associated with sleep pattern and comparison of good sleepers and 
those with sleep problems will show increased BMI which is theoretically related to sleep disorders.

\section{STUdY METHOdology}

During the period of February to May 2015, a cross-sectional survey was planned and conducted with the participation of female students from all the health colleges in Qassim University, KSA. The participants were 288 female students recruited fromhealth Colleges in QassimUniversity (18\% Nursing students, 30.3\% Pharmacy students, $23.6 \%$ Medical students, $16.6 \%$ Applied-Medical-Sciences students and $11.5 \%$ Dental students) and they represent an approximate of $30 \%$ of all undergraduate students in Health Colleges. All enrolled participants were briefed about the purpose of the study and were required to provide signed informed consent before taking part in the study. The study protocol was approved by the Research Center of the College of Nursing, University of Qassim.

\subsection{Study setting}

Qassim city is located in the center of the Arabian Peninsulaand it is the 7th most populated and 5th densest region. The present study was conducted in the female-section, Health colleges in Qassim University, which accommodates students from all provinces and villages of Qassim region. They include Medical, Nursing, Dental, Pharmacy and Applied Medical Sciences students.

\subsection{Tool and data collection procedure}

Data were collected using a self-administered questionnaire which compromised of three parts:

A) Demographic information: Containg questions regardingpersonal data as age, college's name.

B) Anthropometric information: Body Mass Index was calculated based on self-reported body weight $(\mathrm{kg})$ divided by squared height $\left(\mathrm{m}^{2}\right)$. The BMI was then split into four categories: underweight, normal weight, overweightand obesity. According to the WHO, underweight (BMI < 18.5), normal weight (BMI between 18.5-24.9) overweight (BMI between 25-29.9) and obese $(\mathrm{BMI} \pm 30.00) .^{[38]}$

C) Pittsburgh Sleep Quality Index (PSQI) is an effective selfreport instrument used to measure the quality and patterns of sleep for adults. It differentiates "poor" from "good" sleep bymeasuring seven domains: subjective sleep quality, sleep latency, sleep duration, habitual sleep efficiency, sleep disturbances, use of sleep medication, and daytime dysfunction over the last month (see Figure 1). Each of the sleep components yields a score ranging from 0 to 3 , with 3 indicating the greatest dysfunction. Component points range from 0 to 3 and are summed to get a global score, which is from 0 to
21. Highest score suggest greater sleep disturbance; a global score more than 5 suggests a specific disturbance. There are five extra questions have to be completed by a bed partner if there is one. The PSQI has high test-retest reliability and good validity in the psychometric evaluation. ${ }^{[50-52]}$ The present study was utilized for the Arabic version of Pittsburgh Sleep Quality Index, which developed by Suleiman et al., ${ }^{[53]}$ using back translation technique and psychometrically tested in different studies ${ }^{[33,54]}$ which confirmed its validity and reliability for measuring sleep disturbances among Arabic clinical population.

The PSQI consists of three parts:

Part I: It included questions about the personal problems faced by the student during sleep as cold, heat, cough, snoring and waking up to go to the bathroom.

Part II: It contained questions to assess the quality of sleep and some other problems that may disturb sleep.

Part III: Questions on the student's participation in the same room or the same bed for the history of problems noticed during sleep. Data collected was reviewed on a daily basis, and entry process was conducted concurrently with data collection.

\subsection{Data analysis}

Data entry and analysis was done by using SPSS 20. Quantitative variables (Age \& BMI) were given by mean \pm SD. Qualitative variables were shown by using frequency table and percentages. Chi-square test was used to see the relation between PSQI score and body mass index as well as the association between body mass index and sleep duration. $p$-value $<.05$ was taken as significant.

\subsection{Ethical considerations}

The proposal of the investigation was approved by the Research Center of college of Nursing, Qassim University. It was made clear that participant's privacy was to be respected and the study would be anonymous. In addition, participants were given the right to refuse to complete the questionnaire, and the data collected will only be used for scientific purposes. The consent of the students would be sought to participate and that non-participation would not be penalized.

\section{RESULTS}

A total of 288 female University students from different health colleges (Applied Medical Sciences [AMS] 48, Dentistry 33, Medicine 68, Nursing $52 \&$ Pharmacy 87) were involved in the present research. Mean age of all participants was $21.75 \pm 1.86$ years. Mean body mass index of participants was $23.16 \pm 5.03$. As per BMI criteria of WHO, 37 
participants were underweight, 124 participants were normal, ticipants whose sleep quality was good among them, $51.4 \%$ 52 were overweight and 75 were obese (see Table 1). their BMI was normal, $18.9 \%$ were obese and $13.5 \%$ were overweight. Although for both categories good sleep and

\subsection{Participants' sleeping quality and their BMI}

Participants whose sleep quality was poor as per PSQI scores among them $41.8 \%$ their BMI was normal, $27.1 \%$ of participants were obese and $18.7 \%$ were overweight. However parpoor sleep majority of participants their BMI was normal but still no statistically significant association $(p$-value $=.500)$ was seen between BMI and PSQI scores (see Table 2).

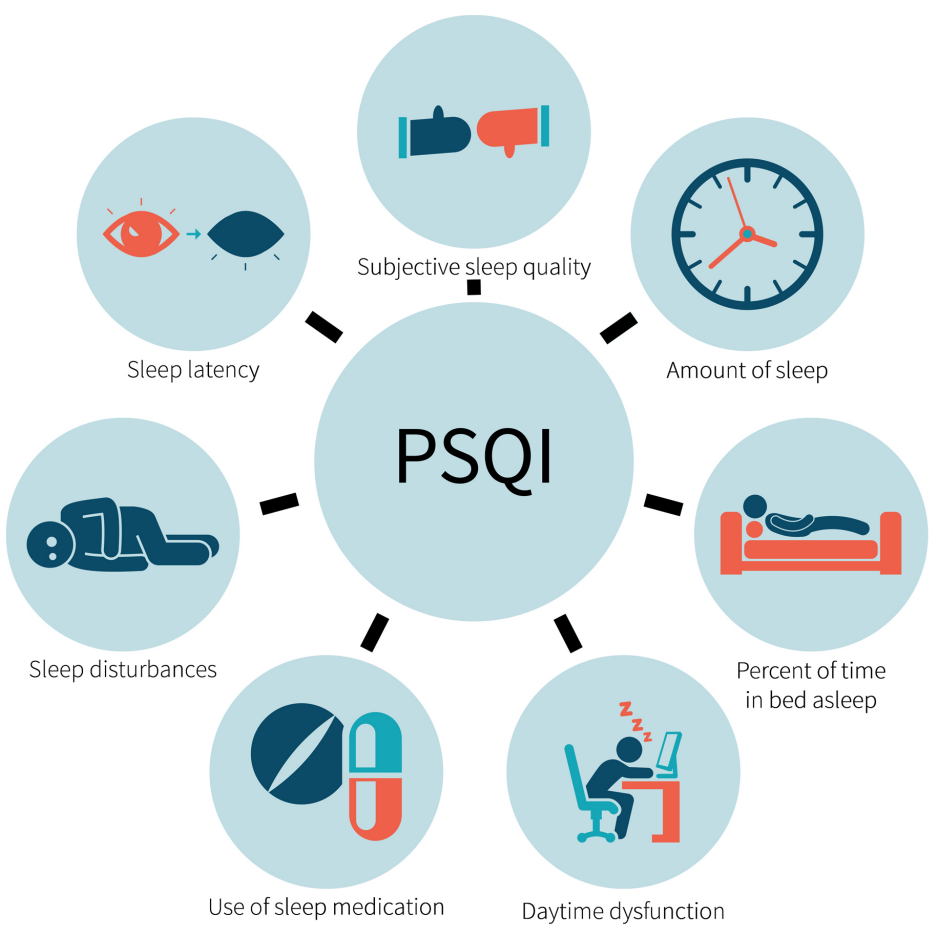

Figure 1. Components of Pittsburgh Sleep Quality Index (PSQI)

Table 1. Mean age and BMI distribution among participants

\begin{tabular}{lllllll}
\hline Health colleges & AMS & Dentistry & Medicine & Nursing & Pharmacy & Total \\
\hline No. & 48 & 33 & 68 & 52 & 87 & 288 \\
Age & $21.50 \pm 1.48$ & $20.91 \pm 1.79$ & $21.03 \pm 1.00$ & $23.42 \pm 2.62$ & $21.76 \pm 1.40$ & $21.75 \pm 1.86$ \\
BMI & $22.91 \pm 4.76$ & $22.13 \pm 3.33$ & $23.09 \pm 6.54$ & $24.03 \pm 5.34$ & $23.23 \pm 4.10$ & $23.16 \pm 5.03$ \\
Under Weight & $3(6.3 \%)$ & $4(12.1 \%)$ & $11(16.2 \%)$ & $9(17.3 \%)$ & $10(11.5 \%)$ & 37 \\
Normal & $26(54.2 \%)$ & $16(48.5 \%)$ & $32(47.1 \%)$ & $13(25 \%)$ & $37(42.5 \%)$ & 124 \\
Over Weight & $9(18.8 \%)$ & $7(21.2 \%)$ & $11(16.2 \%)$ & $11(21.2 \%)$ & $14(16.1 \%)$ & 52 \\
Obese & $10(20.8 \%)$ & $6(18.2 \%)$ & $14(20.6 \%)$ & $19(36.5 \%)$ & $26(29.9 \%)$ & 75 \\
\hline
\end{tabular}

Table 2. Participants' sleeping quality according to their BMI

\begin{tabular}{llll}
\hline \multirow{2}{*}{ BMI } & PSQI Score & & \multirow{2}{*}{ Total } \\
\cline { 2 - 3 } & Good Sleep (<5) & Poor Sleep (> 5) & 37 \\
\hline Under Weight $(<18.5)$ & $6(16.2 \%)$ & $31(12.4 \%)$ & 124 \\
Normal Weight $(18.5-22.99)$ & $19(51.4 \%)$ & $105(41.8 \%)$ & 52 \\
Over Weight $(23-24.9)$ & $5(13.5 \%)$ & $47(18.7 \%)$ & 75 \\
Obese $(>25)$ & $7(18.9 \%)$ & $68(27.1 \%)$ & 288 \\
Total & 37 & 251 & \\
\hline
\end{tabular}

Note. Chi-Square Test $=2.366, p$-value $=.500$. 
3.2 Participants' sleeping quality according to their ferent health colleges. However as per observed trend it was health colleges

Table 3 showed the association between PSQI scores and BMI of participants from different health colleges. As per findings no statistically significant association was detected between PSQI scores and BMI of participants from the difseen that frequency of poor sleep was higher in over weight and obese students from all health colleges but still no statistically significant association was seen between poor sleep and BMI of participants.

Table 3. Participants' sleep quality and BMI according to their health colleges

\begin{tabular}{llllllll}
\hline $\begin{array}{l}\text { Health } \\
\text { College }\end{array}$ & PSQI Score & $\begin{array}{l}\text { Under Weight } \\
(<\mathbf{1 8 . 5})\end{array}$ & $\begin{array}{l}\text { Normal Weight } \\
\mathbf{( 1 8 . 5 - 2 2 . 9 9 )}\end{array}$ & $\begin{array}{l}\text { Over Weight } \\
\mathbf{( 2 3 - 2 4 . 9 )}\end{array}$ & $\begin{array}{l}\text { Obese } \\
(>\mathbf{2 5})\end{array}$ & $\chi^{2}$ Test & $\boldsymbol{p}$-value \\
\hline \multirow{2}{*}{ AMS } & Good Sleep $(\leq 5)$ & $0(0.0 \%)$ & $2(7.7 \%)$ & $1(11.1 \%)$ & $0(0 \%)$ & 1.322 & .721 \\
& Poor Sleep $(>5)$ & $3(100 \%)$ & $24(92.3 \%)$ & $8(88.9 \%)$ & $10(100 \%)$ & & \\
\multirow{2}{*}{ Dentistry } & Good Sleep $(\leq 5)$ & $1(25 \%)$ & $5(31.3 \%)$ & $0(0 \%)$ & $2(33.3 \%)$ & 2.939 & .401 \\
& Poor Sleep $(>5)$ & $3(75 \%)$ & $11(68.8 \%)$ & $7(100 \%)$ & $4(66.7 \%)$ & & \\
\multirow{2}{*}{ Medicine } & Good Sleep $(\leq 5)$ & $2(18.2 \%)$ & $6(18.8 \%)$ & $1(9.1 \%)$ & $0(0 \%)$ & 3.382 & .336 \\
& Poor Sleep $(>5)$ & $9(81.8 \%)$ & $26(81.3 \%)$ & $10(90.9 \%)$ & $14(100 \%)$ & & \\
\multirow{2}{*}{ Nursing } & Good Sleep $(\leq 5)$ & $2(22.2 \%)$ & $2(15.4 \%)$ & $1(9.1 \%)$ & $2(10.5 \%)$ & 0.955 & .812 \\
& Poor Sleep $(>5)$ & $7(77.8 \%)$ & $11(84.6 \%)$ & $10(90.9 \%)$ & $17(89.5 \%)$ & & \\
\multirow{2}{*}{ Pharmacy } & Good Sleep $(\leq 5)$ & $1(10 \%)$ & $4(10.8 \%)$ & $2(14.3 \%)$ & $3(11.5 \%)$ & 0.146 & .986 \\
& Poor Sleep $(>5)$ & $9(90 \%)$ & $33(89.2 \%)$ & $12(85.7 \%)$ & $23(88.5 \%)$ & & \\
\hline
\end{tabular}

3.3 Participants' sleeping pattern and BMI according to their health collegies

For nursing students only the time to fall into sleep has a significantly different in relation to their body mass index. However for other health specialties (AMS, Dentistry, Medicine
\& Pharmacy) no statistically significant differences weredetected in time to fall in sleep in relation to their BMI. As for sleeping hours none of the participants from all specialties show any statistically significant difference in relation to their body mass index (see Table 4).

Table 4. Participants' sleeping pattern and BMI according to their health collegies

\begin{tabular}{|c|c|c|c|c|c|c|c|c|c|c|}
\hline \multirow{3}{*}{ Body Mass Index } & \multicolumn{10}{|c|}{ Q-2: Duration to fall in Sleep } \\
\hline & \multicolumn{2}{|l|}{ AMS } & \multicolumn{2}{|c|}{ Dentistry } & \multicolumn{2}{|c|}{ Medicine } & \multicolumn{2}{|c|}{ Nursing } & \multicolumn{2}{|c|}{ Pharmacy } \\
\hline & Mean & SD & Mean & SD & Mean & SD & Mean & SD & Mean & SD \\
\hline$<18.5$ (Under Weight) & 54.00 & 57.86 & 21.25 & 10.31 & 36.55 & 43.20 & 20.33 & 12.69 & 26.00 & 10.75 \\
\hline 18.5-22.99 (Normal) & 36.73 & 33.67 & 32.69 & 28.61 & 19.53 & 15.47 & 44.23 & 36.16 & 34.24 & 43.88 \\
\hline 23-24.9 (Over Weight) & 21.89 & 16.07 & 45.00 & 35.82 & 23.00 & 18.88 & 20.27 & 11.99 & 28.93 & 28.97 \\
\hline > 25 (Obese) & 35.00 & 34.24 & 23.67 & 21.13 & 23.79 & 18.37 & 48.42 & 32.41 & 28.35 & 17.66 \\
\hline f-test & 0.83 & & 0.89 & & 1.49 & & 3.74 & & 0.27 & \\
\hline$p$-value & .48 & & .46 & & .23 & & $.02 *$ & & .85 & \\
\hline \multirow{3}{*}{ Body Mass Index } & \multicolumn{10}{|c|}{ Q-4: Sleeping Hours } \\
\hline & \multicolumn{2}{|l|}{ AMS } & \multicolumn{2}{|c|}{ Dentistry } & \multicolumn{2}{|c|}{ Medicine } & \multicolumn{2}{|c|}{ Nursing } & \multicolumn{2}{|c|}{ Pharmacy } \\
\hline & Mean & SD & Mean & SD & Mean & SD & Mean & SD & Mean & SD \\
\hline <18.5 (Under Weight) & 6.66 & 3.78 & 5.75 & 0.95 & 6.0 & 2.19 & 7.66 & 2.50 & 7.0 & 2.58 \\
\hline 18.5-22.99 (Normal) & 5.8 & 2.20 & 6.9 & 2.32 & 6.03 & 2.25 & 7.23 & 2.31 & 5.52 & 2.26 \\
\hline 23-24.9 (Over Weight) & 4.83 & 1.06 & 5.14 & 1.34 & 6.45 & 2.54 & 6.0 & 1.56 & 6.39 & 1.44 \\
\hline$>25$ (Obese) & 4.70 & 2.21 & 8.50 & 3.20 & 5.60 & 2.13 & 5.89 & 2.02 & 5.50 & 2.54 \\
\hline f-test & 1.27 & & 2.71 & & 0.29 & & 2.16 & & 1.55 & \\
\hline$p$-value & 0.29 & & 0.06 & & 0.83 & & 0.11 & & 0.21 & \\
\hline
\end{tabular}




\subsection{Sleeping problems among all health colleges' stu- dents in relation to their BMI}

None of the characteristics showed statistically significant difference for body mass index of the participants except among participants from dentistry and college of medicine who wake up at night to use the bathroom and it was significantly associated with body mass index (see Table 5).

In the last month, how frequent have you had sleeping troubles because you

- Q-5a: can't sleep

- Q-5b: wake up during midnight or early morning Q-5c: wakeup for bathroom

- Q-5d: cannot breath

- Q-5e: snoring or coughing loudly
- Q-5f: feel too cold during sleep

- Q-5g: feel too hot during sleep

- Q-5h: had bad dreams

- Q-5i: have pain during sleep

- Q-5j: pain during sleep

\subsection{Sleeping time and components}

According to Table 6, time of sleep was significantly associated with quality of sleep ( $p$-value $=.000)$, actual sleep hours $(p$-value $=.000)$, percentage of time in bed sleeping $(p$-value $=.003)$ and overall sleep disturbance score $(p$-value $=.017)$. No statistically significant association was found for falling a sleep ( $p$-value $=.164)$, using sleeping aid medication $(p$-value $=.921)$ and day time dysfunction $(p$-value $=$ $.122)$.

Table 5. Sleeping problems among health colleges' participants in relation to their BMI

\begin{tabular}{|c|c|c|c|c|c|c|c|c|c|c|c|c|c|c|c|c|c|c|c|c|c|c|c|c|c|c|c|c|c|c|c|c|}
\hline \multirow{2}{*}{$\begin{array}{l}\text { Health } \\
\text { College }\end{array}$} & \multirow{2}{*}{ BMI } & \multicolumn{4}{|c|}{ Q-5a } & \multicolumn{3}{|c|}{ Q-5b } & \multicolumn{3}{|c|}{ Q-5c } & \multicolumn{3}{|c|}{ Q-5d } & \multicolumn{3}{|c|}{ Q-5e } & \multicolumn{3}{|c|}{ Q-5f } & \multicolumn{3}{|c|}{ Q-5g } & \multicolumn{2}{|c|}{ Q-5h } & \multicolumn{3}{|c|}{ Q-5i } & \multicolumn{4}{|c|}{$\mathbf{Q}-5 \mathbf{j}$} \\
\hline & & 0 & 1 & 2 & 3 & 1 & 2 & 3 & 1 & 2 & 3 & 1 & 2 & 3 & 1 & 2 & 3 & 1 & 2 & 3 & 1 & 2 & 3 & 1 & 2 & 3 & 1 & 2 & 3 & 1 & 2 & 3 \\
\hline \multirow{4}{*}{ AMS } & $\begin{array}{l}\text { Under } \\
\text { Weight }\end{array}$ & 0 & 0 & 2 & 1 & 1 & 1 & 1 & 1 & 2 & 0 & 3 & 0 & 0 & 3 & 0 & 0 & 1 & 1 & 1 & 0 & 3 & 0 & 1 & 1 & 1 & 3 & 0 & 0 & 3 & - & 0 \\
\hline & Normal & 6 & 5 & 5 & 10 & 8 & 6 & 12 & 10 & 14 & 2 & 21 & 2 & 3 & 24 & 1 & 1 & 14 & 10 & 2 & 10 & 8 & 8 & 13 & 12 & 1 & 19 & 6 & 1 & 25 & - & 1 \\
\hline & $\begin{array}{l}\text { Over } \\
\text { Weight }\end{array}$ & 1 & 2 & 2 & 4 & 4 & 2 & 3 & 5 & 2 & 2 & 6 & 1 & 2 & 6 & 3 & 0 & 5 & 2 & 2 & 3 & 5 & 1 & 4 & 1 & 4 & 6 & 3 & 0 & 9 & - & 0 \\
\hline & Obese & 1 & 3 & 2 & 4 & 2 & 4 & 4 & 5 & 2 & 3 & 8 & 1 & 1 & 10 & 0 & 0 & 3 & 5 & 2 & 4 & 6 & 0 & 4 & 4 & 2 & 6 & 3 & 1 & 10 & - & 0 \\
\hline$p$-value & & \multicolumn{4}{|c|}{.795} & \multicolumn{3}{|c|}{.907} & \multicolumn{3}{|c|}{.302} & \multicolumn{3}{|c|}{.932} & \multicolumn{3}{|c|}{.124} & \multicolumn{2}{|c|}{.645} & & .1 & & & .13 & & & .80 & & & .83 & & \\
\hline & $\begin{array}{l}\text { Under } \\
\text { Weight }\end{array}$ & 1 & 0 & 2 & 1 & 3 & 1 & 0 & 4 & 0 & 0 & 3 & 0 & 1 & 4 & - & 0 & 3 & 1 & 0 & 3 & 1 & 0 & 2 & 2 & 0 & 4 & 0 & - & 4 & - & 0 \\
\hline Den- & Normal & 4 & 2 & 4 & 6 & 8 & 4 & 4 & 11 & 5 & 0 & 14 & 1 & 1 & 16 & - & 0 & 8 & 3 & 5 & 9 & 4 & 3 & 4 & 9 & 3 & 13 & 3 & - & 14 & - & 2 \\
\hline tistry & $\begin{array}{l}\text { Over } \\
\text { Weight }\end{array}$ & 0 & 3 & 1 & 3 & 4 & 2 & 1 & 5 & 0 & 2 & 4 & 1 & 2 & 7 & - & 0 & 6 & 0 & 1 & 2 & 2 & 3 & 2 & 3 & 2 & 5 & 2 & - & 7 & - & 0 \\
\hline & Obese & 3 & 1 & 0 & 2 & 4 & 1 & 1 & 2 & 4 & 0 & 5 & 1 & 0 & 5 & - & 1 & 4 & 2 & 0 & 2 & 3 & 1 & 4 & 1 & 1 & 6 & 0 & - & 6 & - & 0 \\
\hline$p$-value & & .34 & & & & .9 & 32 & & & & & .5 & & & .21 & & & .3 & & & .5 & & & .53 & & & .40 & & & .52 & & \\
\hline & $\begin{array}{l}\text { Under } \\
\text { Weight }\end{array}$ & 2 & 1 & 4 & 4 & 1 & 3 & 7 & 9 & 2 & 0 & 8 & 2 & 1 & 11 & 0 & - & 3 & 5 & 3 & 5 & 5 & 1 & 6 & 2 & 3 & 11 & 0 & 0 & 11 & - & 0 \\
\hline Medi- & Normal & 8 & 5 & 12 & 7 & 11 & 9 & 12 & 14 & 8 & 10 & 29 & 1 & 2 & 30 & 2 & - & 14 & 14 & 4 & 20 & 7 & 5 & 13 & 14 & 5 & 25 & 4 & 3 & 31 & - & 1 \\
\hline cine & $\begin{array}{l}\text { Over } \\
\text { Weight }\end{array}$ & 3 & 4 & 1 & 3 & 4 & 1 & 6 & 2 & 6 & 3 & 11 & 0 & 0 & 11 & 0 & - & 7 & 3 & 1 & 4 & 5 & 2 & 6 & 4 & 1 & 9 & 2 & 0 & 11 & - & 0 \\
\hline & Obese & 2 & 4 & 4 & 4 & 6 & 2 & 6 & 9 & 4 & 1 & 12 & 1 & 1 & 14 & 0 & - & 9 & 2 & 3 & 7 & 7 & 0 & 5 & 8 & 1 & 9 & 3 & 2 & 14 & - & 0 \\
\hline$p$-value & & .68 & & & & & 50 & & & & & .5 & & & .50 & & & .3 & & & .3 & & & .51 & & & .41 & & & .76 & & \\
\hline & $\begin{array}{l}\text { Under } \\
\text { Weight }\end{array}$ & 1 & 3 & 1 & 4 & 1 & 1 & 7 & 5 & 2 & 2 & 6 & 2 & 1 & 8 & 0 & 1 & 6 & 3 & 0 & 5 & 3 & 1 & 5 & 2 & 2 & 7 & 2 & 0 & 9 & - & 0 \\
\hline Nurcing & Normal & 2 & 4 & 1 & 6 & 4 & 2 & 7 & 5 & 6 & 2 & 10 & 3 & 0 & 13 & 0 & 0 & 8 & 4 & 1 & 6 & 6 & 1 & 7 & 5 & 1 & 10 & 3 & 0 & 13 & - & 0 \\
\hline & $\begin{array}{l}\text { Over } \\
\text { Weight }\end{array}$ & 4 & 1 & 2 & 4 & 2 & 5 & 4 & 5 & 3 & 3 & 8 & 3 & 0 & 10 & 0 & 1 & 4 & 4 & 3 & 3 & 4 & 4 & 6 & 3 & 2 & 7 & 2 & 2 & 11 & - & 0 \\
\hline & Obese & 0 & 3 & 5 & 11 & 1 & 6 & 12 & 6 & 8 & 5 & 14 & 4 & 1 & 18 & 1 & 0 & 12 & 5 & 2 & 9 & 7 & 3 & 8 & 7 & 4 & 15 & 1 & 3 & 18 & - & 1 \\
\hline$p$-value & & .23 & & & & & 32 & & .8 & & & .8 & & & .5 & & & .5 & & & .6 & & & .92 & & & .41 & & & .62 & & \\
\hline & $\begin{array}{l}\text { Under } \\
\text { Weight }\end{array}$ & 3 & 2 & 3 & 2 & 2 & 5 & 3 & 7 & 3 & 0 & 9 & 1 & 0 & 9 & 1 & 0 & 5 & 5 & 0 & 4 & 5 & 1 & 4 & 4 & 2 & 7 & 3 & 0 & 9 & - & 1 \\
\hline Pharma- & Normal & 11 & 12 & 5 & 9 & 10 & 13 & 14 & 18 & 13 & 6 & 29 & 8 & 0 & 37 & 0 & 0 & 20 & 12 & 5 & 20 & 12 & 5 & 14 & 14 & 9 & 23 & 12 & 2 & 37 & - & 0 \\
\hline cy & $\begin{array}{l}\text { Over } \\
\text { Weight }\end{array}$ & 3 & 5 & 3 & 3 & 7 & 2 & 5 & 5 & 4 & 5 & 12 & 2 & 0 & 13 & 0 & 1 & 10 & 3 & 1 & 6 & 5 & 3 & 7 & 4 & 3 & 11 & 2 & 1 & 13 & - & 1 \\
\hline & Obese & 4 & 5 & 6 & 11 & 5 & 9 & 12 & 14 & 7 & 5 & 21 & 4 & 1 & 25 & 1 & 0 & 17 & 9 & 0 & 10 & 8 & 8 & 14 & 9 & 3 & 20 & 5 & 1 & 26 & - & 0 \\
\hline$p$-value & & .64 & & & & .3 & 77 & & .4 & & & .7 & & & .15 & & & .3 & & & .5 & & & .85 & & & .77 & & & .13 & & \\
\hline
\end{tabular}

\section{Discussion}

The present study was designed to explore the associations between sleep patterns and BMI among undergraduate female students in health colleges. Our study adds to the literature the inclusion of a sample 288 of undergraduate female health colleges' students, their sleep pattern has been measured by Pittsburgh Sleep Quality Index (PSQI), which was used to assess sleep quality of participants and its association with Body Mass Index which measured by $\left(\mathrm{KG} / \mathrm{M}^{2}\right)$. Research searching the link between sleep and BMI among 
adolescents, suggest that the relationship is complex. ${ }^{[55,56]}$ Most previous studies have shown an inverse association between BMI and sleep difficulty and duration. ${ }^{[4,57,58]}$ Even with the different age group as Soares et al., ${ }^{[48]}$ who con- ducted his study among university students or specific gender as Xinag et al., ${ }^{[49]}$ who targeted females rather than males from the general population in China.

Table 6. Sleeping time and components among participants

\begin{tabular}{|c|c|c|c|c|c|c|}
\hline \multirow{2}{*}{ Sleep Components } & & \multicolumn{2}{|l|}{ Sleeping Time } & \multirow{2}{*}{ Total } & \multirow{2}{*}{$\begin{array}{l}\text { Chi-Square } \\
\text { Test }\end{array}$} & \multirow{2}{*}{$p$-value } \\
\hline & & Before or at $12 \mathrm{Pm}$ & After 12 Pm & & & \\
\hline \multirow{4}{*}{ Quality of sleep } & Very Good & $33(61.1 \%)$ & $21(38.9 \%)$ & 54 & \multirow{4}{*}{30.12} & \multirow{4}{*}{.000} \\
\hline & Fairly Good & $55(49.5 \%)$ & $56(50.5 \%)$ & 111 & & \\
\hline & Fairly Bad & $16(20 \%)$ & $64(80 \%)$ & 80 & & \\
\hline & Very Bad & $12(27.9 \%)$ & $31(72.1 \%)$ & 43 & & \\
\hline \multirow{4}{*}{ Falling sleep } & $\leq 15 \mathrm{~min}$ & $49(45 \%)$ & $60(55 \%)$ & 109 & \multirow{4}{*}{5.10} & \multirow{4}{*}{.164} \\
\hline & $16-30 \mathrm{~min}$ & $48(42.5 \%)$ & $65(57.5 \%)$ & 113 & & \\
\hline & $31-60 \mathrm{~min}$ & $15(30.6 \%)$ & $34(69.4 \%)$ & 49 & & \\
\hline & $>60 \mathrm{~min}$ & $4(23.5 \%)$ & $13(76.5 \%)$ & 17 & & \\
\hline \multirow{4}{*}{ Actual Sleep Hours } & $>7$ hours & $48(48 \%)$ & $52(52 \%)$ & 100 & \multirow{4}{*}{26.97} & \multirow{4}{*}{.000} \\
\hline & $6-7$ hours & $28(54.9 \%)$ & $23(45.1 \%)$ & 51 & & \\
\hline & 5-6 hours & $29(45.3 \%)$ & $35(54.7 \%)$ & 64 & & \\
\hline & $<5$ hours & $11(15.1 \%)$ & $62(84.9 \%)$ & 73 & & \\
\hline \multirow{4}{*}{$\begin{array}{l}\text { Percentage oftime } \\
\text { in bed sleeping }\end{array}$} & $>85 \%$ & $75(35.4 \%)$ & $137(64.6 \%)$ & 212 & \multirow{4}{*}{14.04} & \multirow{4}{*}{.003} \\
\hline & $75 \%-84 \%$ & $8(36.4 \%)$ & $14(63.6 \%)$ & 22 & & \\
\hline & $65 \%-74 \%$ & $14(63.6 \%)$ & $8(36.4 \%)$ & 22 & & \\
\hline & $<65 \%$ & $17(65.4 \%)$ & $9(34.6 \%)$ & 26 & & \\
\hline \multirow{4}{*}{$\begin{array}{l}\text { Take medicine to } \\
\text { aid in sleep }\end{array}$} & Not in past month & $106(40.9 \%)$ & $153(59.1 \%)$ & 259 & \multirow{4}{*}{0.492} & \multirow{4}{*}{.921} \\
\hline & $<$ once a week & $6(33.3 \%)$ & $12(66.7 \%)$ & 18 & & \\
\hline & 1-2 times a week & $3(37.5 \%)$ & $5(62.5 \%)$ & 8 & & \\
\hline & $\geq 3$ times a week & $1(33.3 \%)$ & $2(66.7 \%)$ & 3 & & \\
\hline \multirow{4}{*}{$\begin{array}{l}\text { Daytime } \\
\text { dysfunction }\end{array}$} & 0 & $9(52.9 \%)$ & $8(47.1 \%)$ & 17 & \multirow{4}{*}{5.796} & \multirow{4}{*}{.122} \\
\hline & $1-2$ & $51(44.3 \%)$ & $64(55.7 \%)$ & 115 & & \\
\hline & $3-4$ & $45(39.8 \%)$ & $68(60.2 \%)$ & 113 & & \\
\hline & $5-6$ & $11(25.6 \%)$ & $32(74.4 \%)$ & 43 & & \\
\hline \multirow{4}{*}{$\begin{array}{l}\text { Overall sleep } \\
\text { disturbance score }\end{array}$} & $1-4$ & $13(56.5 \%)$ & $10(43.5 \%)$ & 23 & \multirow{4}{*}{10.22} & \multirow{4}{*}{.017} \\
\hline & $5-8$ & $63(47 \%)$ & $71(53 \%)$ & 134 & & \\
\hline & $9-12$ & $36(30.5 \%)$ & $82(69.5 \%)$ & 118 & & \\
\hline & $>12$ & $4(30.8 \%)$ & $9(69.2 \%)$ & 13 & & \\
\hline
\end{tabular}

One study showed that short self-reported sleep duration is linked to elevated BMI and may change appetite hormones $^{[47]}$ and in addition, a very recent study by Peltzer and Pengpid ${ }^{[59]}$ confirmed an association between short sleep duration and increased BMI. In 2010, Lombardi et al. ${ }^{[45]}$ reported that poor quality or decreased sleep has been associated with obesity, this study's assumption supported by our observed finding; frequency of poor sleep was higher in over weight and obese participants as rate of $45.8 \%$ from all participants but still no statistically significant association was seen between poor sleep and body mass index of participants within the PSQI scoring. Otherwise, sleep quality and duration to fall into sleep in relation with BMI-as a single question-found nursing students only had statically association between sleep quality/duration to fall in sleep and BMI. Also, we found participants from dentistry and medicine colleges who were waking up at night to use bathroom was significantly related with body mass index. In contrast, sleep difficulties at baseline are associated with current and longtime lower BMI, ${ }^{[27,47]}$ but the inverse association is not con- 
firmed. Lower BMI is not a significant predictor of sleep difficulties over time ${ }^{[60]}$ Report on the association of sleep and BMI in the clinical sample and in community samples are mixed and do not show a consistent association between sleep patterns and BMI. ${ }^{[61]}$ However, the inverse association between sleep difficulties and $\mathrm{BMI}^{[49,62]}$ is unexpected considering the findings of most studies on sleep restriction and weight/obesity which revealed that short/long sleep and weight increase/obesity are associated. ${ }^{[62,63]}$ There are some proofs that sleep education programs and cognitive behavior interventions can improve sleep in college students. ${ }^{[65]}$

The current study has restrictions that should be addressed. First the study was designed as an exploratory study depending on a reasonably limited evidence showing that continues disturbanceof sleep can produce increased body weights by altering metabolic function. Our investigation was based on self-reported data including sleep period rather than direct measurements. BMI values were also limited to self-report, rather than actual weight and height measures or more sophisticated assessments of adiposity. However, although individuals generally underreport weight and overreport height, the correspondence between anthropometric and self-report measures tend to be high (generally above .94; e.g. ${ }^{64,65]}$ ) and it is unlikely that small inaccuracies in BMI would have dramatically impacted our correlational findings. Second, we have not measured students' level of daily physical activity or dietary intake. Third, in addition to measurement limitations, the characteristics of the research entrants-college students-limit the generalizabilityof our results to the general population. The nature of the study precludes conclusions about the real relationship between sleep disturbances and BMI. Finally, we cannot rule out the possibility that our results are due to other extraneous variables or to chance. Future researches would benefit from collecting different data points prospectively using objective measurements of obesity and sleep.

\section{Conclusion}

The results of our study suggested that there's no association between sleep pattern and body mass index among undergraduate female health colleges' students at Qassim University, according to PSQI. As well, a suggested high prevalence of poor sleep quality. Finally, we recommended regulating of eating behavior, daily activity, stress and activate time management in order to improve sleep quality.

\section{ACKNOWLEDGEMENTS}

The authors gratefully acknowledge that all undergraduate female students who so willingly participated in this study. As well the Research Center of College of Nursing that approved the proposal and tool for data collection. We thank the administration of all health colleges who facilitated the recruitment of subjects and collection of data and also we are grateful to the other members that made this study possible.

\section{CONFLicts OF InTEREST Disclosure}

No potential conflicts of interest with respect to the research, authorship, and publication of this article.

\section{REFERENCES}

[1] Ruthig J, Haynes T, Stupnisky R, et al. Perceived Academic Control: mediating the effects of optimism and social support on college students' psychological health. Soc Psychol Educ. 2009; 12(2): 233-49. https://doi.org/10.1007/s11218-008-9079-6

[2] Gaultney JF. The prevalence of sleep disorders in college students: impact on academic performance. J Am Coll Health. 2010; 59(2): 91-7. PMid:20864434 https://doi .org/10.1080/07448481.2 010.483708

[3] Lund HG, Reider BD, Whiting AB, et al. Sleep Patterns and Predictors of Disturbed Sleep in a Large Population of College Students. J Adolesc Health. 2010; 46(2): 124-32. PMid:20113918 https://doi.org/10.1016/j.jadohealth.2009.06.016

[4] Sing CY, Wong WS. Prevalence of Insomnia and Its Psychosocial Correlates Among College Students in Hong Kong. J Am Coll Health. 2010; 59(3): 174-82. PMid:21186447 https ://doi .org/10.108 $0 / 07448481.2010 .497829$

[5] Nadorff MR, Nazem S, Fiske A. Insomnia symptoms, nightmares, and suicidal ideation in a college student sample. Sleep. 2011; 34(1): 93. PMid:21203379 https ://doi .org/10.1093/sleep/ 34.1 .93

Published by Sciedu Press
[6] Nyeret M, Farabaugh A, Fehling K, et al. Relationship Between Sleep Disturbance and Depression, Anxiety, and Functioning in College Students. Depress Anxiety. 2013; 00: 1-8.

[7] Cirelli C, Tononi G. Is sleep essential? PLoS Biol. 2008; 6: e216. PMid:18752355 https://doi.org/10.1371/journal.pbio.0 060216

[8] Siegel JM. Sleep viewed as a state of adaptive inactivity. Nat Rev Neurosci. 2009; 10: 747-753. PMid:19654581 https://doi .org/ $10.1038 / \mathrm{nrn} 2697$

[9] Brand S, Kirov R. Sleep and its importance in adolescence and in common adolescent somatic and psychiatric conditions. International Journal of General Medicine. 2011; 4 425-442. PMid:21731894 https://doi.org/10.2147/IJGM.S11557

[10] Diekelmann S, Born J. The memory function of sleep. Nat Rev Neurosci. 2010; 11: 114-126. https://doi.org/10.1038/nrn2762

[11] Krueger JM, Rector DM, Roy S, et al. Sleep as a fundamental property of neuronal assemblies. Nat Rev. Neurosci. 2008; 9: 910-919. PMid:18985047 https://doi.org/10.1038/nrn2521

[12] Kim HN, Cho J, Chang Y, et al. Association between Personality Traits and Sleep Quality in Young Korean Women. PLOS ONE. 
2015; 10(6): e0129599. https://doi.org/10.1371/journal. pone. 0129599

[13] Boyle PJ, Scott JC, Krentz AJ, et al. Diminished brain glucose metabolism is a significant determinant for falling rates of systemic glucose utilization during sleep in normal humans. J Clin Invest. 1994; 93(2): 529. PMid:8113391 https://doi .org/10.1172/JC I117003

[14] Scheen AJ, Byrne MM, Plat L, et al. Relationships between sleep quality and glucose regulation in normal humans. American J PhysiolEndocrinol Metab. 1996; 34(2): E261. PMid:8770019 https : //do i.org/10.1152/ajpendo.1996.271.2.E261

[15] Markwald RR, Melanson EL, Smith MR, et al. Impact of insufficient sleep on total daily energy expenditure, food intake, and weight gain. Proc Natl Acad Sci. 2013; 110(14): 5695-5700. PMid:23479616 https://doi.org/10.1073/pnas. 1216951110

[16] Tasali E, Leproult R, Ehrmann DA, et al. Slow-wave sleep and the risk of type 2 diabetes in humans. Proc Natl Acad Sci USA. 2008; 105(3): 1044-9. PMid:18172212 https://doi .org/10.1073/pn as .0706446105

[17] Seicean S, Kirchner HL, Gottlieb DJ, et al. Sleep-Disordered Breathing and Impaired Glucose Metabolism in Normal-Weight and Overweight/Obese Individuals The Sleep Heart Health Study. Diabetes Care. 2008; 31(5): 1001-1006. PMid:18268072 https://doi.or $\mathrm{g} / 10.2337 / \mathrm{dc} 07-2003$

[18] Al-Disi D, Al-Daghri N, Khanam L, et al. Subjective sleep duration and quality influence diet composition and circulating adipocytokines and ghrelin levels in teen-age girls. Endoc J. 2010; 57(10): 915-23. https://doi.org/10.1507/endocrj.K10E-145

[19] Stamatakis KA, Punjabi NM. Effects of Sleep Fragmentation on Glucose Metabolism in Normal Subjects. Chest. 2010; 137(1): 95-101. PMid:19542260 https://doi.org/10.1378/chest.09-0791

[20] Kong AP, Wing YK, Choi KC, et al. Associations of sleep duration with obesity and serum lipid profile in children and adolescents. Sleep Med. 2011; 12(7): 659-65. PMid:21689984 https: //doi.org/10.1016/j.sleep.2010.12.015

[21] Lucassen EA, Rother KI, Cizza G. Interacting epidemics? Sleep curtailment, insulin resistance, and obesity. Ann N Y Acad Sci. 2012; 1264(1): 110-34. PMid:22827862 https://doi.org/10.1111/j. 1749-6632.2012.06655.x

[22] Gangwisch JE, Malaspina D, Babiss LA, et al. Short sleep duration as a risk factor for hypercholesterolemia: analyses of the National Longitudinal Study of Adolescent Health. Sleep. 2010; 33(7): 956-61. PMid:20614855 https://doi .org/10.1093/sleep/33.7.956

[23] Lesser DJ, Bhatia R, Tran WH, et al. Sleep fragmentation and intermittent hypoxemia are associated with decreased insulin sensitivity in obese adolescent Latino males. Pediatr Res. 2012; 72(3): 293-8 PMid:22669298 https://doi.org/10.1038/pr.2012.73

[24] Shankar A, Syamala S, Kalidindi S. Insufficient rest or sleep and its relation to cardiovascular disease, diabetes and obesity in a national, multiethnic sample. PLoS One. 2010; 5(11): e14189. PMid:21152066 https://doi.org/10.1371/journal. pone.0 014189

[25] Morris CJ, Yang JN, Scheer FA. The impact of the circadian timing system on cardiovascular and metabolic function. Prog Brain Res. 2012; 199: 337-58. https://doi.org/10.1016/B978-0-444-5 9427-3.00019-8

[26] Roenneberg T, Allebrandt KV, Merrow M, et al. Social jetlag and obesity. Curr Biol. 2012; 22(10): 939-43. PMid:22578422 https : //doi.org/10.1016/j.cub.2012.03.038

[27] Soares MJ, Macedo A, Bos S, et al. Sleep disturbances, body mass index and eating behavior in undergraduate students. J Sleep Res.
2011; 20(3): 479-86. PMid:20887393 https://doi.org/10.111 $1 / j .1365-2869.2010 .00887 . x$

[28] Knutson KL, Van Cauter E, Rathouz PJ, et al. Trends in the prevalence of short sleepers in the USA: 1975-2006. Sleep. 2010; 33(1): 37. PMid:20120619 https://doi.org/10.1093/sleep/33.1.37

[29] Franklin K, Lindberg, E. Obstructive sleep apnea is a common disorder in the population-a review on the epidemiology of sleep apnea. Journal of Thoracic Disease. 2015; 7(8): 1311-1322.

[30] Cote AT, Harris KC, Panagiotopoulos C, et al. Childhood obesity and cardiovascular dysfunction. J Am Coll Cardiol. 2013; 62(15): 13091319. PMid:23954339 https://doi .org/10.1016/j . jacc. 201 3.07 .042

[31] Lieberman J, Neubauer D. Normal Sleep and Wakefulness. Int J Sleep Wakefulness-Prim Care. 2007; 1(1): 2-6.

[32] Giri PA, Baviskar MP, Phalke DB. Study of Sleep Habits and Sleep Problems among Medical Students of Parvara Institute of Medical Sciences loni, western Maharashtra, India. Ann Med Health Sci Res. 2013; 3: 51-4. PMid:23634330 https://doi.org/10.4103/21 41-9248. 109488

[33] Krueger P, Friedman EM. Sleep duration in the United States: A cross-sectional population-based study. American Journal of Epidemiology. 2009; 169: 1052-1063. PMid:19299406 https ://doi . org/10.1093/aje/kwp023

[34] Mahfouz M, Ageely H, Alsaruri S, et al. Sleep Quality among students of the factuality of medicine Jazan University, Saudi Arabia. Middle-East Journal of Scientific Research. 2013; 16(4): 508-513.

[35] Ogden CL, Carroll MD, Kit BK, et al. Prevalence of obesity and trends in body mass index among U.S. children and adolescents, 1999-2010. JAMA. 2012; 307(5): 483-90. PMid:22253364 https : //doi.org/10.1001/jama.2012.40

[36] Fryar CD, Carroll MD, Ogden CL. Prevalence of Overweight Obesity, Extreme Obesity Among Adults: United States, Trends 1960-1962 Through 2009-2010. Centers for Disease Control and Prevention (CDC). 2012. Available from: http://198.246.124.29/nchs/data/hestat/obesity_ad ult_09_10/obesity_adult_09_10.pdf

[37] Flegal KM, Arll MD, Ogden CL, et al. Prevalence and trends in obesity among us adults, 1999-2008. JAMA. 2010; 303(3): 235-41. PMid:20071471 https://doi.org/10.1001/jama.2009.2014

[38] World Health Organization. Obesity and overweight. 2016. Available from: http://www.who.int/mediacentre/factsheets /fs311/en/

[39] Al-Nohair S. Obesity in Gulf Countries. International Journal of Health Sciences. 2014; 8(1): 79-83. https ://doi .org/10.12816 10006074

[40] Al-Islam M, Faris S, Abu Jamous D, et al. Assessing obesity, Body fatness and dietary behaviors among adult College Students in Hail, SA. International Journal of Nutrition and Food Sciences. 2014; 3(2): 60-68. https://doi.org/10.11648/j.ijnfs. 20140302.17

[41] Huang TTK, Harris KJ, Lee RE, et al. Assessing overweight, obesity, diet, and physical activity in college students. J Am Coll Health. 2003; 52(2): 83-6. PMid:14765762 https ://doi .org/10.1080/ 07448480309595728

[42] American College Health Association. American College Health Association-National College. Hanover, MD: 2012. Health Assessment II: Reference Group Summary Report. Spring 2012. Available from: http://www. acha-ncha.org/docs/ACHA-NCHA-II_Re ferenceGroup_DataReport_Spring2012.pdf [Accessed June 12, 2014].

[43] Vargas PA, Flores M, Robles E. Sleep Quality and Body Mass Index in College Students: The Role of Sleep Disturbances. J Am Coll 
Health. 2014; 62(8): 534-541. https://doi.org/10.1080/0744 8481.2014 .933344

[44] American Psychiatric Association. Diagnostic and statistical manual of mental disorders. 5th ed. Washington DC: American Psychiatric Association; 2013.

[45] Lombardi D, Wirtz A, Willetts J, et al. Independent Effects of Sleep Duration and Body Mass Index on the Risk of a Work-Related Injury: Evidence From the US National Health Interview Survey. The Journal of Biological and Medical Rhythm Research. 2012; 29(5): 556-564.

[46] Wal J. Night eating syndrome: a critical review of the literature. Clinical Psychology Review. 2012; 32: 49-59. PMid:22142838 https://doi.org/10.1016/j.cpr.2011.11.001

[47] Bjorvatn B, Sagen IM, Qyane N, et al. The association between sleep duration, body mass index and metabolic measures in the Hordaland Health Study. Journal of Sleep Research. 2007; 16(1): 66-76. PMid:17309765 https://doi .org/10.1111/j.1365-2869.20 $07.00569 . \mathrm{x}$

[48] Soares MJ, Macedo A, Azevedo M. Sleep disturbances and eating behaviors in undergraduate students. In handbook of nutrition, diet and sleep. UK: wageningen academic publishers; 2013.

[49] Xiang Y, Ma X, Lu J, et al. Relationship of sleep duration with sleep disturbances, basic socio-demographic factors and BMI in Chinese people. Sleep Medicine. 2009; 10: 1085-1089. PMid:19442580 https://doi.org/10.1016/j.sleep.2009.03.002

[50] Fichtenberg NL, Putnam SH, Mann NR, et al. Insomnia Screening in Postacute Traumatic Brain Injury: Utility and Validity of the Pittsburgh Sleep Quality Index. Am J Phys Med Rehabil. 2001; 80(5). https://doi.org/10.1097/00002060-200105000-00003

[51] Backhaus J, Junghanns K, Broocks A, et al. Test-retest reliability and validity of the Pittsburgh Sleep Quality Index in primary insomnia. Journal of Psychosomatic Research. 2002; 53(3): 737-40. https://doi.org/10.1016/S0022-3999(02)00330-6

[52] Spira AP, Beaudreau SA, Stone KL, et al. Reliability and validity of the Pittsburgh sleep quality index and the Epworth sleepiness scale in older men. J Gerontol A Biol Sci Med Sci. 2012; 67(4): 433-439. https://doi.org/10.1093/gerona/glr172

[53] Suleiman K, Yates B, Berger A, et al. Translating the Pittsburgh Sleep Quality Index into Arabic. Western Journal of Nursing Research. 2010; 32(2): 250-268. PMid:19915205 https://doi.org/ 10.1177/0193945909348230

[54] Suleiman K, Al-Hadid L, Duhni A. Psychometric Testing of the Arabic version of the Pittsburgh Sleep Quality Index (A-PSQI) among Coronary Artery Disease Patients in Jordan. Journal of Natural Sciences Research. 2012; 2(8): 15-19.
[55] Knutson KL. The association between pubertal status and sleep duration and quality among a nationally representative sample of U.S Adolescents. Am J Hum Biol. 2005; 17: 418-424. PMid:15981178 https://doi.org/10.1002/ajhb. 20405

[56] Garaulet M, Ortega FB, Ruiz JR, et al. Short sleep duration is associated with increased obesity markers in European adolescents: effect of physical activity and dietary habits. The Helena study. Int J Obes. 2011; 35: 1308-1317. PMid:21792170 https://doi .org/10.103 8/ijo.2011.149

[57] Najafian J, Mohammadifard N, Siadat ZD, et al. Association between Sleep Duration and Body Mass Index and Waist Circumference. Iran J Med Sci. June 2010.

[58] Benham G. The Association Between Body Mass Index and Sleep in a Predominantly Hispanic College Population. Hispanic Journal of Behavioral Sciences. 2017; 39(3): 389-397. https ://doi .org/10 $.1177 / 0739986317707721$

[59] Peltzer K, Pengpid S. Sleep Duration, Sleep Quality, Body Mass Index, and Waist Circumference among Young Adults from 24 Lowand Middle-Income and Two High-Income Countries. Int J Environ Res Public Health. June 2017; 14(6): 566. PMid:28587107 https://doi.org/10.3390/ijerph14060566

[60] Bos SC, Soares MJ, Marques M, et al. Disorder eating behaviors and sleep disturbances. Eating Behaviors. 2013; 14: 192198. PMid:23557819 https://doi.org/10.1016/j. eatbeh.2 013.01 .012

[61] Soares MJ, Macedo A. Modulation of sleep by obesity, diabetes, age and diet. University of Arizona. Tucson: 2014.

[62] Cappuccio F, Taggart F, Kandala NB, et al. Meta-analysis of short sleep duration abd obesity in children and adult. Sleep. 2008; 31: 619626. PMid:18517032 https://doi.org/10.1093/sleep/31.5 .619

[63] Gangwisch J, Malaspina D, Boden-albala B, et al. Inadequate sleep as a risk factor for obesity: analysis of the NHANES I. Sleep. 2005; 28: 1289-1296. PMid:16295214 https://doi.org/10.1093/s1 eep/28.10.1289

[64] Jackowska M, Brown J, Ronaldson A, et al. The impact of a brief gratitude intervention on subjective well-being, biology and sleep. Journal of Health Psychology. 2016; 21: 2207-2217. https: //doi.org/10.1177/1359105315572455

[65] Pursey K, Burrows TL, Stanwell P, et al. How accurate is webbased self-reported height, weight, and body mass index in young adults? Journal of Medical Internet Research. 2014; 16(1): e4. https://doi.org/10.2196/jmir.2909 